\title{
Thermal response of skin diseased tissue treated by plasmonic nanoantenna
}

\author{
Rasha H. Mahdi, Hussein A. Jawad \\ Institute of Laser for Postgraduate Studies, University of Baghdad, Iraq
}

\begin{tabular}{l} 
Article Info \\
\hline Article history: \\
Received Jul 11, 2019 \\
Revised Jan 4, 2020 \\
Accepted Jan 12, 2020 \\
\hline
\end{tabular}

\section{Keywords:}

Bowtie

Optical nanoantenna

Plasmonics

SAR

Temperature distribution

\begin{abstract}
The thermal distribution in the diseased tissue treated by different methods faces the problem of an uncontrollable defused heat. In the present article, we use a plasmonic bowtie nanoantenna working in the near infrared region to enhance the temperature confinement in the tissue. The Computer Simulation Technology Studio Suite package version 2019 was used to execute the design of both plasmonic nanoantenna and the tissue. Gold nanostructure and silicon carbide dioxide are the components the plasmonic nanoantenna in the bowtie shape. The results showed that the distance between the tumor tissue and the antenna is important to determine the intensity field where the maximum field is $5.9^{*} 10^{7} \mathrm{~V} / \mathrm{m}$ at a distance of $100 \mathrm{~nm}$. The maximum specific absorption rate is $1.92 * 10^{11} \mathrm{~W} / \mathrm{kg}$ at a similar distance which gives a higher temperature in the tissue of $580 \mathrm{C}^{\circ}$. It is concluded that from the obtained results that the near infrared $(1064 \mathrm{~nm})$ resonance wavelength is recommended in the treatment of cancer cell by plasmonic bowtie nanoantenna because higher intensity field is generated. The closer distance to the nanoantenna gives higher temperature in the tissue while the temperature gradually decreases in the tissue till $400 \mathrm{~nm}$ where no valuable temperature was detected.
\end{abstract}

Copyright @ 2020 Institute of Advanced Engineering and Science. All rights reserved.

\section{Corresponding Author:}

Rasha H. Mahdi,

Institute of Laser for Postgraduate Studies,

University of Baghdad,

Baghdad, Iraq.

Email: rasha@ilps.uobaghdad.edu.iq

\section{INTRODUCTION}

The interaction of light with metal nanostructure is the backbone of plasmonics. The metal nanostructure is frequency dependent. The designed nano-structures are well known to generate the hot-spots where the incident field is enhanced by several order of magnitude. The plasmonic resonance observed in these structures opens the ability to design antennas operating in the optical spectrum [1,2].

Optical nanoantennas of the nano-sized metallic particle can improve the mismatch between the diffraction limited spot of the excitation light and fluorescent molecules that are much smaller than the incident wavelength [3]. Confinement of light on subwavelength scales with large local fields by the exploitation of surface plasmons, which are collective charge oscillations, leads the demonstration of revolutionary concepts [4]. Plasmonic nanoantenna can produce very high near field intensities due to their localized surface plasmon resonance (LSPR) [2]. Different applications are depending on near field enhancement generated by plasmonic optical nanoantennas such as surface-enhanced Raman spectroscopy (SERS), biosensing, cancer treatment, spectral imaging, solar applications, and near-field probes [5, 6].

Among various categories of nanostructures reported so far to manipulate LSPR, bowtie nanoantenna holds a unique position due to its giant electric field enhancement at the sharp metal tips and nanofocusing characteristics [7, 8]. Hence bowtie nanostructures have been used for high-performance plasmonic applications [9]. The well-known example of nanoantenna is the bowtie antenna, where 
a subwavelength air gap between two metallic regions can enhance the electric field more than 100 times [10, 11].

The size, shape, and morphology of the nanostructure could be engineered, in addition to the interparticle distance and dielectric environment [12], their LSPRs can be tuned from visible to the NIR region $(700-1400 \mathrm{~nm})$. The near-infrared region (NIR region) is the so-called biological window which is particular interest for deep-tissue imaging and treatment [13]. Light to heat conversion is employed for photothermal therapy and photothermal drug release in cancer cells [14] The gold nanostructures, especially with plasmonic properties suitable into the NIR region, show great promise for cancer photothermal therapy through nonradiative photothermal effect, demonstrating the ability to destroy cancerous lesion [15], Key features to consider when selecting a nanostructure for photothermal therapy are the plasmon resonance wavelength, the absorption cross-section, and the size of the nanoparticle $[14,16]$.

The optical properties of the skin determine light distribution in the irradiated region (epidermis, dermis and subcutaneous) [17]. The optical technologies promise less danger to the patient [18]. Seriously research is done to investigate and analyze heat transfer through the skin tissue to obtain the temperature distribution but the heat confinement still a challenge. In this work, we design both plasmonic bowtie nanoantenna working at $1046 \mathrm{~nm}$ and the proposed tumor tissue using Computer Simulation Technology (CST) software. Specific Absorption Rate (SAR) and temperature distribution in the tumor could be estimated.

\section{MATERIALS AND METHODS}

The plasmonic nanoantenna was designed via CST Studio Suite packaged version 2019. The materials used are gold and $\mathrm{SiO}_{2}$. The resonance wavelength of the designed nanoantenna is $1064 \mathrm{~nm}$. Two design wear accomplished, one for plasmonic bowtie and the other for the proposed tumor tissue. The details of the design are illustrated in the following subsections.

\subsection{Plasmonic bowtie design}

A three-dimensional (3D) plasmonic bowtie nanoantennas working at resonance wavelength $1064 \mathrm{~nm}$ was designed. It is composed of a metal/dielectric. The used real and imaginary parts of the gold dielectric function with respect to different incident wavelengths are based on experimental data [19]. The refractive index of the $\mathrm{SiO}_{2}$ substrate is 1.5 [20]. The bowtie structure is normally illuminated by linearly polarized waveguide excitation source along the $\mathrm{x}$-axis (x-polarization). The surrounding environment of the design structure is assumed to be air.

The sweeping process was done to select suitable geometric parameters of the designed structure to get the required resonance wavelength [21]. The results are antenna length and width ( $\mathrm{L}=\mathrm{W}=294 \mathrm{~nm})$, thickness $(T=60 \mathrm{~nm})$, the apex width $(\mathrm{A}=20 \mathrm{~nm})$, the gap width $(\mathrm{G}=20 \mathrm{~nm})$ and the bowtie angle $\left(\Theta=90^{\circ}\right)$, and the length, the width, and the thickness of the $\mathrm{SiO}_{2}$ substrate was set as $(700,700$, and 200) $\mathrm{nm}$ respectively, as shown in Figure 1.

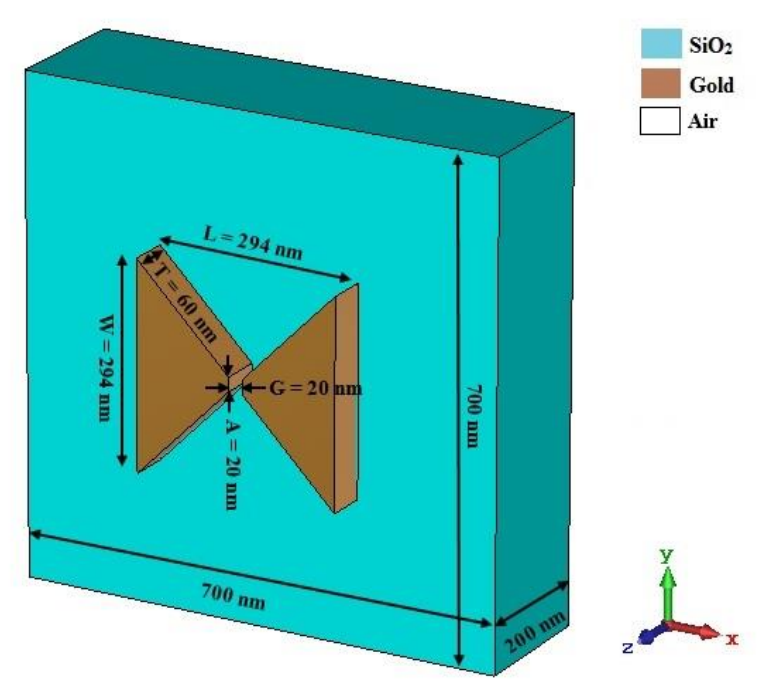

Figure 1. 3D Schematic view of the plasmonic bowtie nanoantenna 


\subsection{Tissue model}

The proposed tumor tissue is the skin. The position of the tumor tissue is in the center of the skin structure. The dimensions of skin are selected in relation with the bowtie structure dimensions where the tissue length and width are $(\mathrm{L}=\mathrm{W}=600 \mathrm{~nm})$ while the thickness is $(\mathrm{T}=300 \mathrm{~nm})$. A spherical shape of the tumor tissue of $(\mathrm{D}=100 \mathrm{~nm})$ in diameter is proposed as shown in Figure 2.

The thermal properties of the tissue are listed in Table 1. In addition, the dielectric properties of the tissue play an important role in the investigation of the propagation characteristic of the plasmonic optical nanoantenna. These properties are mainly depending on tissue type and the wavelength of interest. The designed tissue is subjected to the plasmonic nanoantenna radiation at different distances, the resulted pattern is shown in Figure 3.

Table 1. The properties of the tissues [22]

\begin{tabular}{llll}
\hline Tissue & Thermal Conductivity $\mathrm{K}(\mathrm{W} / \mathrm{m})$ & Specific HeatC $(\mathrm{kJ} / \mathrm{K} / \mathrm{kg})$ & Mass Density $\rho(\mathrm{kg} / \mathrm{m} 3)$ \\
\hline Skin & 0.2 & 3.6 & 1200 \\
tumor & 0.5 & 3.6 & 1050 \\
\hline
\end{tabular}

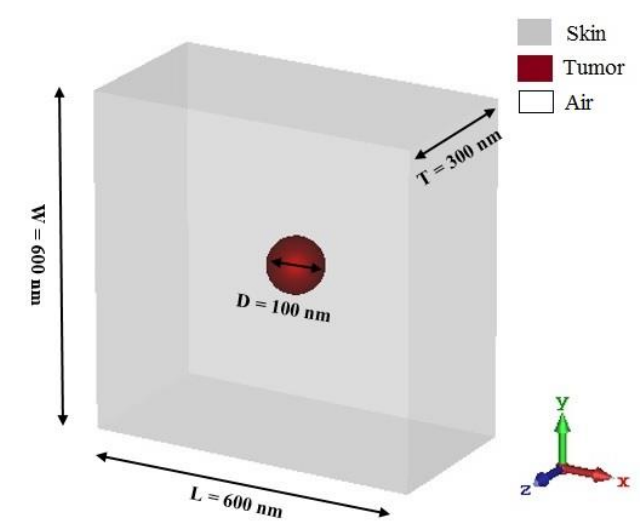

Figure 2. 3D Schematic view of the tumor embedded in the skin tissue

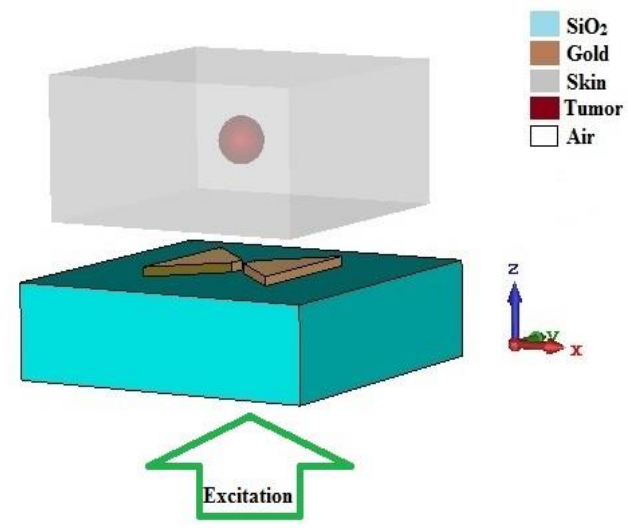

Figure 3. The final pattern of bowtie nanoantenna in front of the designed tissue

\section{RESULTS AND ANALYSIS}

The performance of the plasmonic bowtie, SAR calculations and temperature estimation in the tissue are investigated and analyzed.

\subsection{Performance of plasmonic bowtie nanoantenna}

The reflectivity in $\mathrm{dB}$ as a function of the wavelength is shown in Figure 4. The maximum reflectivity is detected at the wavelength of $1064 \mathrm{~nm}$ where it reaches $-27.70 \mathrm{~dB}$. The length of nanoantenna plays an important role in the shifted resonance wavelength. Figure 5 shows that the electric field is highly confined in the gap of the bowtie structure. The enhanced near electric field is $2 * 10^{8} \mathrm{~V} / \mathrm{m}$ and the gap width 
of $20 \mathrm{~nm}$ expected. The far electric field in three dimensions for the antenna at different distances $(100,200,300$, and 400) nm, was investigated as shown in Figure 6 (a, b, c, and d). The estimated far field $\left(5.9 * 10^{7}, 2.95 * 10^{7}, 1.97 * 10^{7}\right.$ and $\left.1.47 * 10^{7}\right) \mathrm{V} / \mathrm{m}$ at distances $(100,200,300$, and 400$) \mathrm{nm}$, respectively.

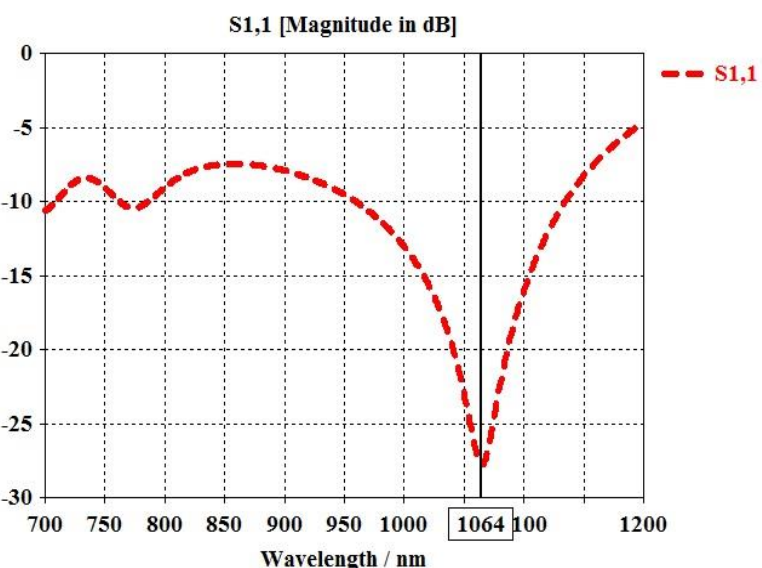

Figure 4. The reflectivity of plasmonic bowtie nanoantenna at $1064 \mathrm{~nm}$

(a)

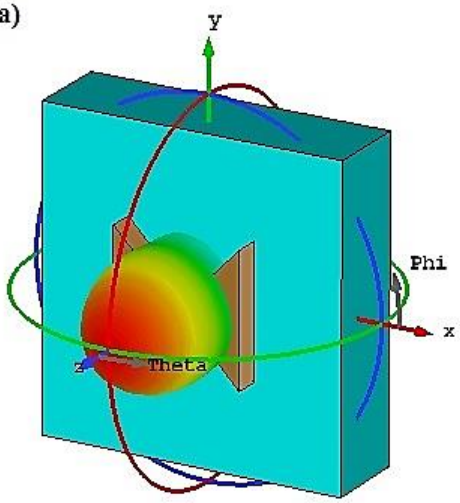

(c)

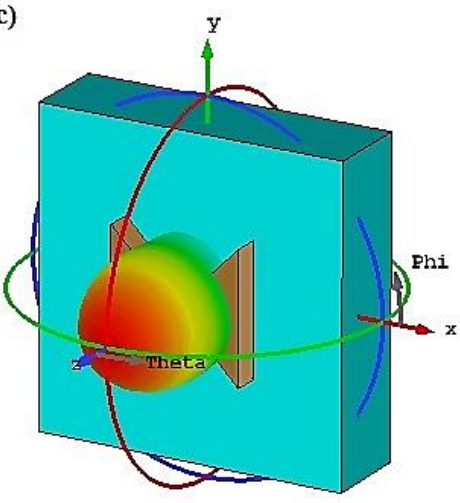

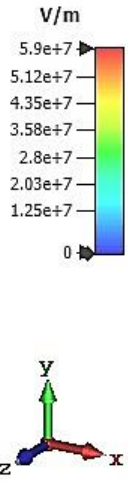

$\mathrm{v} / \mathrm{m}$

$1.97 \mathrm{e}+7 \gg$

$1.71 \mathrm{e}+7$

$1.45 e+7$

$1.19 \mathrm{e}+7$

$9.34 \mathrm{e}+6-$

$6.75 e+6$

$4.17 \mathrm{e}+6-$

$0 \rightarrow$
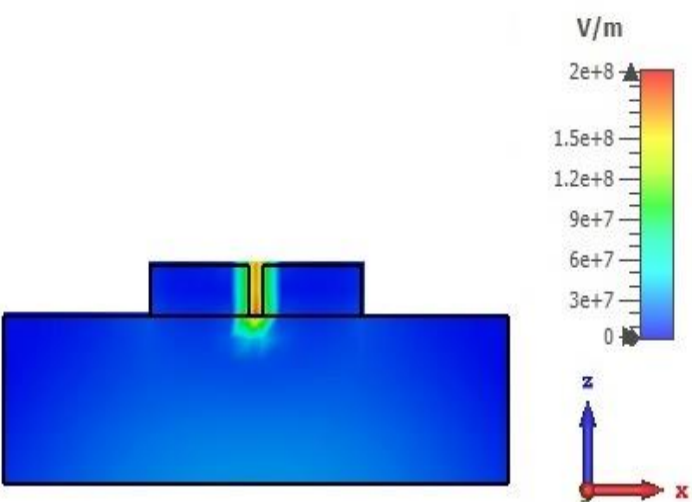

Figure 5. Electric field distribution at the LSPR frequency of plasmonic bowtie nanoantenna at a gap of $20 \mathrm{~nm}$

(b)

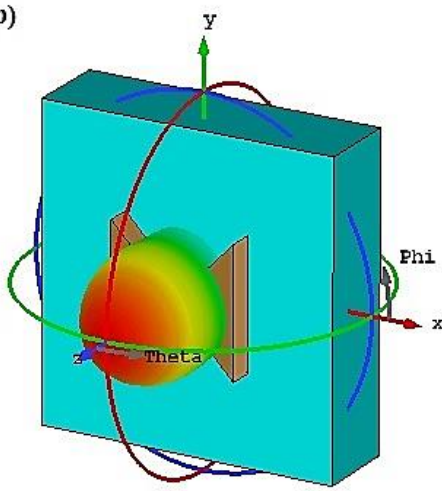

$\mathrm{V} / \mathrm{m}$

$2.95 \mathrm{e}+7$

$2.56 \mathrm{e}+7-$

$2.18 \mathrm{e}+7$

$1.01 \mathrm{e}+7$

$6.26 \mathrm{e}+6-$

0 a

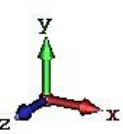

(d)

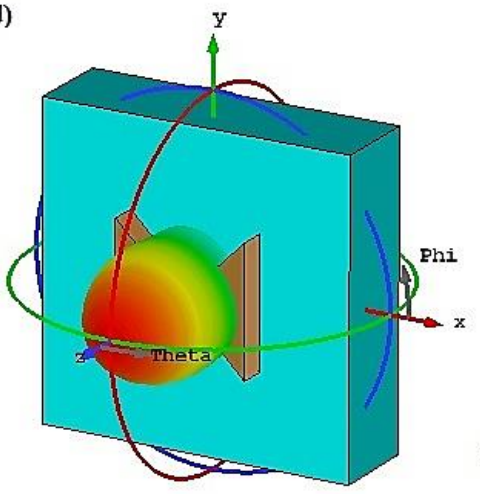

$\mathrm{v} / \mathrm{m}$

$1.47 \mathrm{e}+7$

$1.28 \mathrm{e}+7$ $1.09 \mathrm{e}+7-$

$7 e+6-$

507 e+6

0.

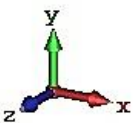

Figure 6. The far-field pattern of nanoantenna at different distances;

(a) $100 \mathrm{~nm}$, (b) $200 \mathrm{~nm}$, (c) $300 \mathrm{~nm}$, (d) $400 \mathrm{~nm}$ 


\subsection{SAR calculation}

SAR is a measure of electromagnetic power density and subsequent absorption of electromagnetic radiation and conversion to heat by human tissue. It is expressed as [23].

$$
\mathrm{SAR}=\sigma|\mathrm{E}|^{2} / 2 \rho
$$

where:

$\sigma=$ the conductivity of the tissue-simulating material $(\mathrm{S} / \mathrm{m})$

$\mathrm{E}=$ the total RMS field strength $(\mathrm{V} / \mathrm{m})$

$\rho=$ the mass density of tissue-simulating material $\left(\mathrm{kg} / \mathrm{m}^{3}\right)$

The resonance wavelength $1064 \mathrm{~nm}$ was detected for the design nanoantenna alone but when the proposed tissue is exposed to the antenna at different distances, the estimated SAR values could be varied due to the variation in the reflectivity and hence in the far field pattern. Now, the irradiated tissue by bowtie nanoantenna at different distances $(100,200,300$, and 400) $\mathrm{nm}$ is shown in Figure 7 . The variation in the reflectivity of the designed antenna is studied for every distance. Where the reflectivity measured is $(-15.33,-19.71,-27.57$, and -43.84$) \mathrm{dB}$ for the distances $(100,200,300$, and 400$) \mathrm{nm}$ respectively. It is observed that the detected reflectivity is red shift starting from $100 \mathrm{~nm}$. The maximum wavelength shift was detected at a distance of 400 and may be reached to the resonance wavelength at a certain distance as in Figure 8. The far field distribution in the proposed tissue at different distances from the nanoantenna was investigated where the maximum far-field measured is $\left(6.87 * 10^{7}, 3.14^{*} 10^{7}, 2.42^{*} 10^{7}\right.$, and $\left.2.15^{*} 10^{7}\right) \mathrm{V} / \mathrm{m}$ for the distances $(100,200,300$, and 400) $\mathrm{nm}$ respectively. The far-field pattern for every distance is shown in Figure 9, which indicated that the far-field decreased as the distance increased.

(a)

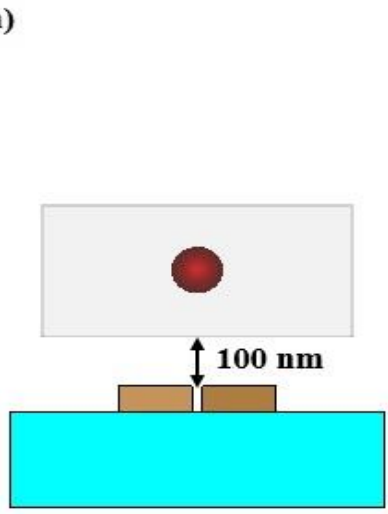

(c)
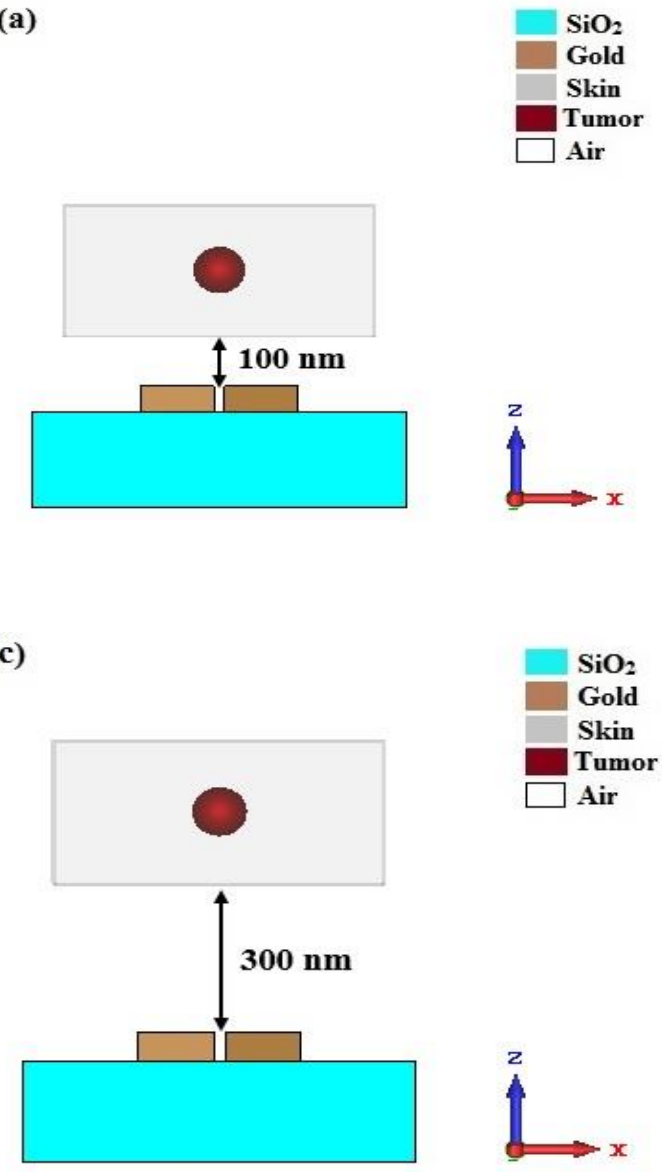

(b)

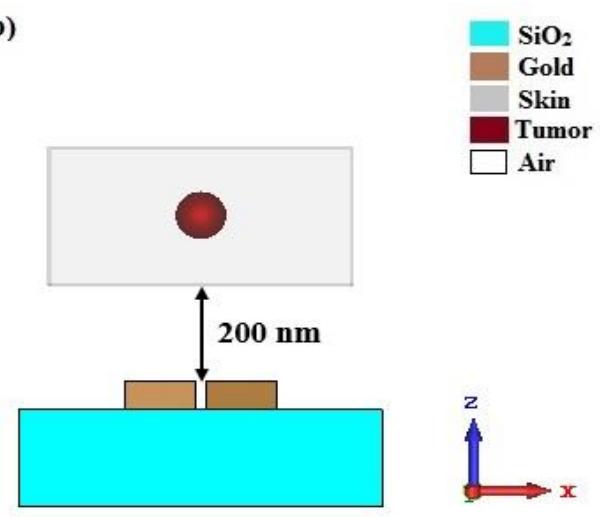

(d)

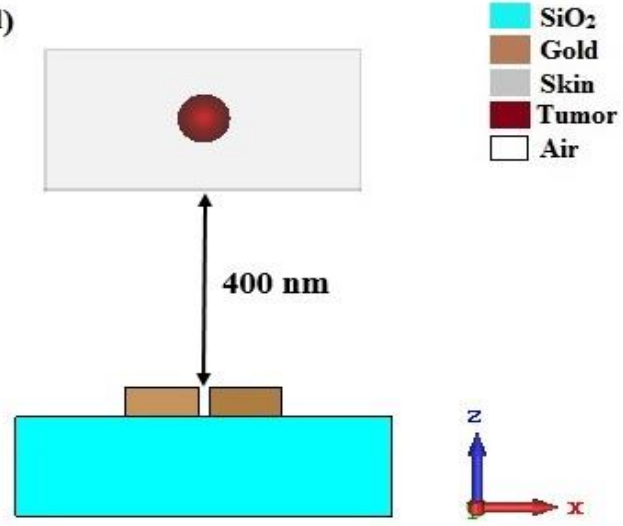

Figure 7. Side view of the proposed tissue exposed to plasmonic bowtie nanoantenna at different distances; (a) $100 \mathrm{~nm}$, (b) $200 \mathrm{~nm}$, (c) $300 \mathrm{~nm}$, (d) $400 \mathrm{~nm}$ 

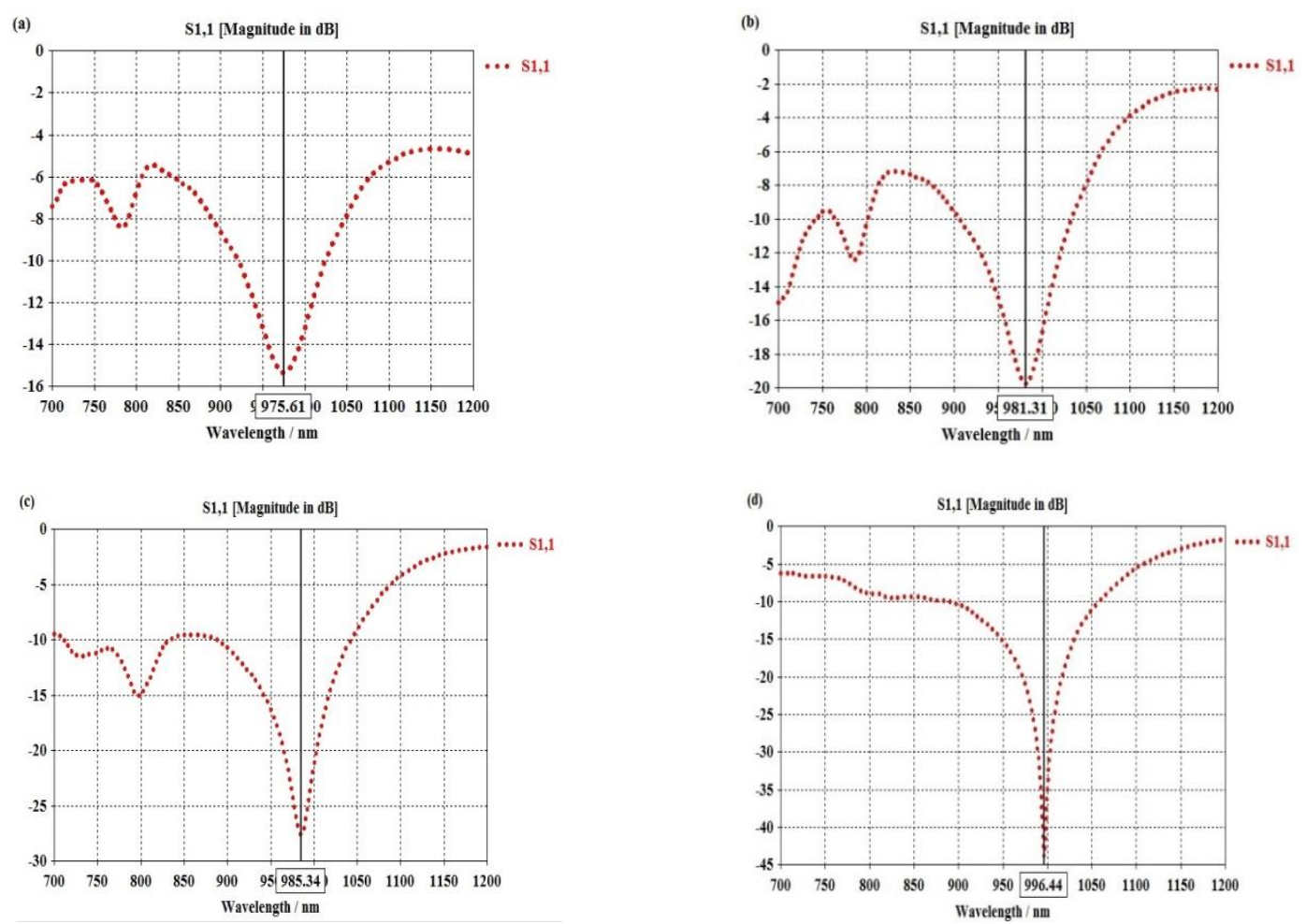

Figure 8 . The reflectivity of plasmonic bowtie nanoantenna at different distances from the tissue; (a) $100 \mathrm{~nm}$, (b) $200 \mathrm{~nm}$, (c) $300 \mathrm{~nm}$, (d) $400 \mathrm{~nm}$

(a)

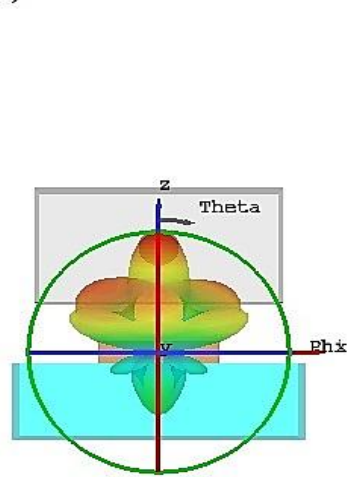

(c)
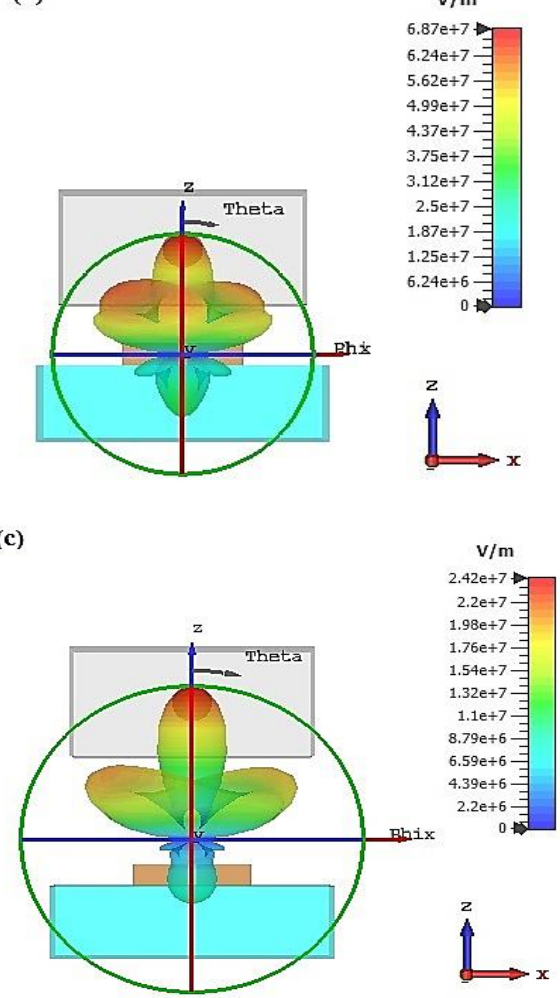

(b)
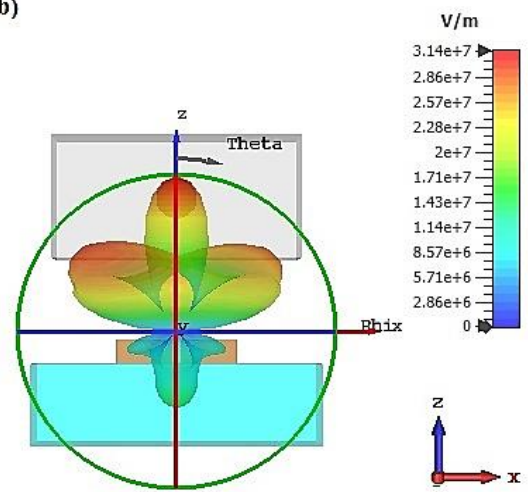

(d)

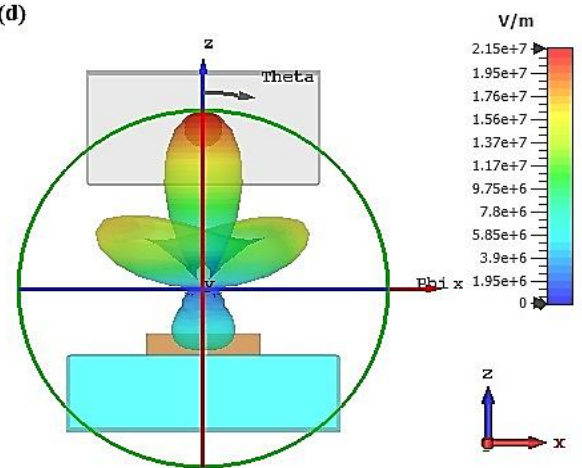

Figure 9. The far-field pattern in the proposed tissue at different distances; (a) $100 \mathrm{~nm}$, (b) $200 \mathrm{~nm}$, (c) $300 \mathrm{~nm}$, (d) $400 \mathrm{~nm}$ 
After the reflectivity and the far-field pattern are studied, the point SAR inside the proposed tissue could be estimated. As $\left(1.92 * 10^{11}, 8.76 * 10^{10}, 6.23 * 10^{10}, 6.13 * 10^{10}\right)$ at distances $(100,200,300$, and 400$) \mathrm{nm}$ respectively. The calculated SAR at different distances is observed in Figure 10. It seems that the calculated $\mathrm{SAR}$ is decreased with distance.

(a)

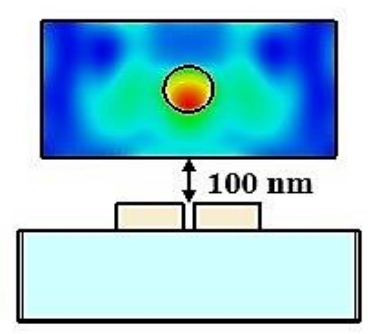

(c)

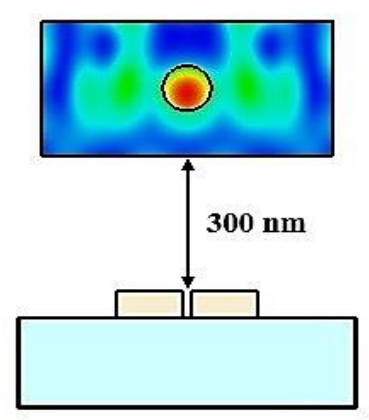

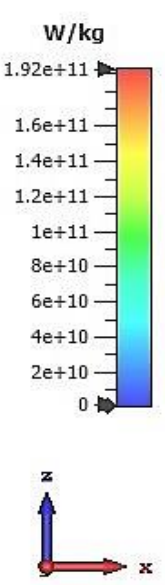

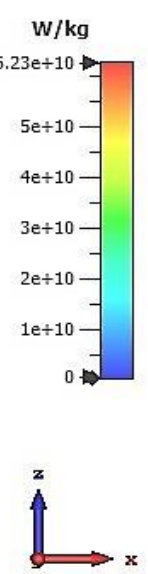

(b)

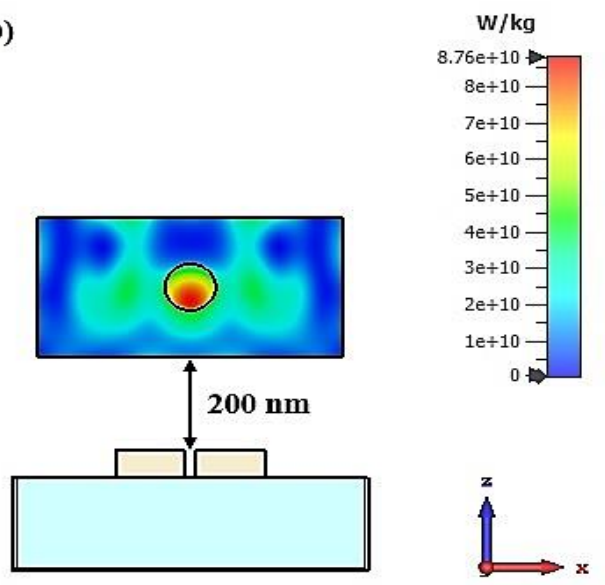

(d)

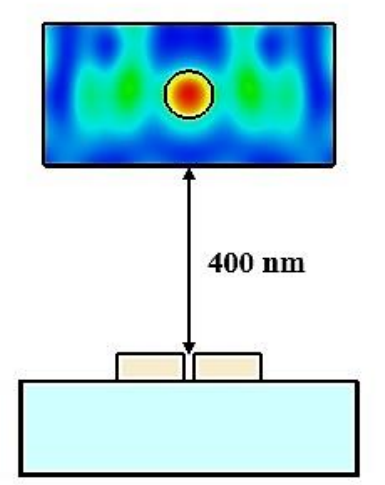

$\mathrm{w} / \mathrm{kg}$

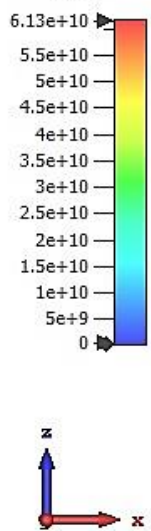

Figure 10. The calculated point SAR in the proposed tissue at different distances from the antenna; (a) $100 \mathrm{~nm}$, (b) $200 \mathrm{~nm}$, (c) $300 \mathrm{~nm}$, (d) $400 \mathrm{~nm}$

\subsection{Temperature calculation}

The absorption of power from electromagnetic fields causes a temperature rise in tissues. The high levels of absorbed power can cause irreversible tissue damage. The temperature could be calculated from the SAR estimation values related to (2) [24].

$$
\Delta \mathrm{T}=(\mathrm{SAR} \times \Delta \mathrm{t}) / \mathrm{C}
$$

where:-

$\mathrm{T}=$ the temperature in Kelvin $(\mathrm{K})$

$\Delta \mathrm{t}=$ the time period

$\mathrm{C}=$ the specific heat $(\mathrm{J} / \mathrm{K} . \mathrm{kg})$

From the obtained results of point SAR, the temperature distribution in the tumor could be calculated. The estimated temperature variation in the proposed tissue is $580 \mathrm{C}^{\circ}$ at $100 \mathrm{~nm}, 116 \mathrm{C}^{\circ}$ at $200 \mathrm{~nm}, 4 \mathrm{C}^{\circ}$ at $300 \mathrm{~nm}$ and $0 \mathrm{C}^{\circ}$ at $400 \mathrm{~nm}$ respectively. 


\section{DISCUSSION}

The optical plasmonic nanoantenna proves high ability to destruct the tumor tissues especially the cancer cells due to these antennas are regarded as a hot point source which means that the desired tissue could be destroyed without affecting surrounding healthy tissues. Our purpose is to estimate the thermal distribution in the tissue at different distances from the nanoantenna. The designed of plasmonic nanoantenna working in near-infrared is directly influenced by different parameters (the shape, the length, and the gap dimension) in addition to the material used where the resonance wavelength plays an important role in the field enhancement. In recent work, the bowtie shape of plasmonic nanoantenna is selected because the sharp tips of the bowtie antenna, the group and phase velocities of surface plasmonic waves decrease with the distance of propagation and finally become zero at the sharp tips of the bowtie [25]. The generated field could be enhanced several order of magnitude in the gap, so the gap width should be selected accurately. The maximum near field is generated at the resonance wavelength. The gold metal is the best choices in the plasmonic structure in the medical applications for different reasons, non-toxic material, anti oxidized and working at resonance wavelength $530 \mathrm{~nm}$. The optical wavelength gives a higher intensity field which repressed a good source to treat the tumor cells. The dielectric material $\left(\mathrm{SiO}_{2}\right)$ is selected to affect the wavelength shift in the resonance wavelength. It is worth mentioned here that the interaction of the incident field with nanoantennas is a nonlinear interaction. so, we usually expected that the two-photon absorption occurs because of the resonance wavelength in the NIR region, the resonance wavelength of the gold cannot be obtained unless the two-photon absorption takes place. The resonance wavelength is obtained after different tries where the affected parameters (thickness, gap, length, width, flare angle) are varied many times. The more effective parameter in the design of bowtie nanoantenna is the gap width. It is useful to mention that the variation of every parameter effects directly on the others so, the compromisation between those parameters should be regarded. The tumor tissue dimensions are designed related to the bowtie nanoantenna taken in to account three main factors the position of the tumor inside the skin, the location of the tumor in the skin human body and the distance between nanoantenna and the tumor cells. It is observed that the distance effects on the field reaching to the tissue where the field reduced when the distance increased which means the temp distribution in the tissue is decreased as seen later. The SAR calculation for different cases is important to estimate the temperature distribution in the tissue. The calculated results of the SAR proved that the best value of the SAR is the closest to the tissue which causes to the higher temperature generated in the tissue, knowing that the resonance wavelength is varied related to every distance and doesn't represent the best. The calculated SAR in the waveguide which is used in this research is higher than used by [26]. which proves that NIR resonance wavelength is better than millimeter wave region that generates less SAR.

The main goal of the current research work is to estimate the thermal distribution in the tissue which is investigated through the temperature calculation in the tissue and the reaction of the tissue against plasmonic bowtie nanoantenna. The estimated pattern of far field distribution in the tissue showed that the distribution of far field is varied depending on the distance from the antenna. The maximum temperature generated in the tissue under the effect of plasmonic bowtie nanoantenna is detected at a distance of $100 \mathrm{~nm}$, which gives a clear indication that the distance from bowtie nanoantenna Influences on the distribution field in the tissue and hens on the generated temperature.

\section{CONCLUSION}

The temperature distribution in the tumor tissue attracted many researchers due to its effect on the destruction of the cancer cells. The field confinement generated by plasmonic bowtie nanoantenna was the main tool to destruct the cancer cells leaving the healthy tissue unaffected. From the obtained results, we can conclude that: the NIR $(1064 \mathrm{~nm})$ resonance wavelength of plasmonic bowtie nanoantenna is recommended in the treatment of cancer cell because higher intensity field is generated. The closer distance to the nanoantenna gives higher temperature in the tissue which regards over the allowed increasing temperature in the tissue.

\section{ACKNOWLEDGEMENTS}

We appreciate the efforts of the Institute of Laser for Postgraduate Studies, University of Baghdad, Baghdad-Iraq, to support the research work. 


\section{REFERENCES}

[1] D. P. Fromm, A. Sundaramurthy, P. J. Schuck, G. Kino, and W. E. Moerner, "Gap-Dependent optical coupling of single 'bowtie' nanoantennas resonant in the visible," Nano Lett., vol. 4, no. 5, pp. 957-961, May 2004.

[2] P. Mühlschlegel, H.-J. Eisler, O. J. F. Martin, B. Hecht, and D. W. Pohl, "Resonant optical antennas," Science, vol. 308, no. 5728, pp. 1607-1609, Jun. 2005.

[3] L. Rogobete, F. Kaminski, M. Agio, and V. Sandoghdar, "Design of plasmonic nanoantennae for enhancing spontaneous emission," Opt. Lett., vol. 32, no. 12, pp. 1623-1625, Jun. 2007.

[4] A. E. Cetin, S. Aksu, M. Turkmen, D. Etezadi, and H. Altug, "Theoretical and experimental analysis of subwavelength bowtie-shaped antennas," J. Electromagn. Waves Appl., vol. 29, no. 13, pp. 1686-1698, Sep. 2015.

[5] B. B. Yousif and A. S. Samra, "Modeling of optical nanoantennas," Physics Research International, Phys. Res. Int., vol. 2012, pp. 10, 2012.

[6] R. M. Taha and H. A. Jawad, "Characterization of gold coating on nano structure CR39 polymer as SERS sensor," Iraqi J. of laser part A, vol. 17, 2018.

[7] P. J. Schuck, D. P. Fromm, A. Sundaramurthy, G. S. Kino, and W. E. Moerner, "Improving the mismatch between light and nanoscale objects with gold bowtie nanoantennas," Phys. Rev. Lett., vol. 94, no. 1, pp. 017402, Jan. 2005.

[8] N. Yu, E. Cubukcu, L. Diehl, D. Bour, S. Corzine, J. Zhu, G. Höfler, K. B. Crozier, and F. Capasso, "Bowtie plasmonic quantum cascade laser antenna," Opt. Express, vol. 15, no. 20, pp. 13272-13281, Oct. 2007.

[9] D. Hasan, C. P. Ho, P. Pitchappa, and C. Lee, "Dipolar resonance enhancement and magnetic resonance in crosscoupled bow-tie nanoantenna array by plasmonic cavity," ACS Photonics, vol. 2, no. 7, pp. 890-898, Jul. 2015.

[10] D. K. Gramotnev, A. Pors, M. Willatzen, and S. I. Bozhevolnyi, "Gap-plasmon nanoantennas and bowtie resonators," Phys. Rev. B, vol. 85, no. 4, pp. 045434, Jan. 2012.

[11] P. B. Johnson and R. W. Christy, "Optical constants of the noble metals," Phys. Rev. B, vol. 6, no. 12, pp. 4370-4379, Dec. 1972.

[12] J. Jeevanandam, A. Barhoum, Y. S. Chan, A. Dufresne, and M. K. Danquah, "Review on nanoparticles and nanostructured materials: history, sources, toxicity and regulations," Beilstein J. of nanotechnol, vol. 9, pp.1050-1074, Apr. 2018.

[13] L. A. Sordillo, S. Pratavieir, Y. Pu, K. S.-Ramirez, L. Shi, L. Zhang, Y. Budansky, R. R. Alfano, "Third therapeutic spectral window for deep tissue imaging," The International Society for Optical Engineering, vol. 89400, pp. 89400V, Mar. 2014.

[14] Y. Gao and Y. Li, "Gold nanostructures for cancer imaging and therapy," in Advances in Nanotheranostics I: Design and Fabrication of Theranosic Nanoparticles, Z. Dai, Ed. Berlin, Heidelberg: Springer Berlin Heidelberg, pp. 53-101, 2016.

[15] L. C. Kennedy, L. R. Bickford, N. A. Lewinski, A. J. Coughlin, Y. Hu, E. S. Day, J. L. West, and R. A. Drezek, "A new era for cancer treatment: gold-nanoparticle-mediated thermal therapies," Small Weinh Bergstr Ger., vol. 7, no. 2, pp. 169-183, Jan. 2011.

[16] O. Brzobohaty, M. Siler, J. Trojek, L. Chvatal, V. Karasek, A. Patak, Z. Pokorna, F. Mika and P. Zemanek, "Three-dimensional optical trapping of a plasmonic nanoparticle using low numerical aperture optical tweezers," Sci. Rep., vol. 5, pp. 8106, Jan. 2015.

[17] A. N. Bashkatov, E. A. Genina and V. V. Tuchin, "A review: optical properties of skin, subcutaneous, and muscle tissues,” J. of Innovative Opt. Health Sciences, vol. 4, no. 1, pp. 9-38, Jan. 2011.

[18] A.N. Bashkatov, E.A. Genina, V.I. Kochubey, and V.V. Tuchin, "Optical properties of human skin, subcutaneous and mucous tissues in the wavelength range from 400 to 2000 ไhspace0.167emnm," J. Phys. Appl. Phys., vol. 38, no. 15, pp. 2543-2555, Jul. 2005.

[19] Y. F. Chau, H. H. Yeh, and D. P. Tsai, "A new type of optical antenna: plasmonics nanoshell bowtie antenna with dielectric hole,” J. Electromagn. Waves Appl., vol. 24, no. 11, pp. 1621-1632, Jul. 2010.

[20] M. A. Gatea and H.A. Jawad, "Thermoplasmonic of single Au@SiO2 and SiO2@Au core shell nanoparticles in deionized warte and poly-vinylpyrrolidone matrix," Baghdad Science J., vol. 16, no. 2, 2019.

[21] M.K.A. Rahim, M.Z.A. Abdul Aziz and C.S. Goh, "Bow-tie microstrip antenna design," 13th IEEE International Conference on Networks Jointly held with the 2005 IEEE 7th Malaysia International Conf on Communic, Kuala Lumpur, pp. 4, 2005.

[22] K. Shurrab, N. Kochaji, and W. Bachir, "Development of temperature distribution and light propagation model in biological tissue irradiated by $980 \mathrm{~nm}$ laser diode and using comsol simulation," J. Lasers Med. Sci., vol. 8, no. 3, pp. 118-122, Jun. 2017.

[23] B. M. Hochwald, D. J. Love, S. Yan, and J. Jin, "SAR codes," in Information Theory and Applications Workshop (ITA), pp. 1-9, 2013.

[24] A. H. Sallomi, "A theoretical approach for SAR calculation in human head exposed to RF signals," J. of Eng. and Dev., vol. 16, no. 4, pp. 304-313, Dec. 2012.

[25] G. Jayaswal, A. Belkadi, A. Meredov, B. Pelz, G. Moddel and A. Shamim, "A Zero-Bias, completely passive 28 $\mathrm{THz}$ rectenna for energy harvesting from infrared (waste heat)," IEEE/MTT-S Int. Microw. Symp.-IMS, pp. 355-358, 2018.

[26] T. Hamed and M. Maqsood, "SAR calculation \& temperature response of human body exposure to electromagnetic radiations at 28, 40 and $60 \mathrm{GHz}$ mm wave frequencies," Prog. Electromagn. Res. M, vol. 73, pp. 47-49, 2018. 